\title{
Determination of Raman emission cross-section ratio in hydrogenated microcrystalline silicon
}

\author{
E. Vallat-Sauvain *, C. Droz, F. Meillaud, J. Bailat, A. Shah, C. Ballif \\ Institute of Microtechnology, University of Neuchâtel, rue Breguet 2, CH-2000 Neuchâtel, Switzerland
}

Available online 17 April 2006

\begin{abstract}
The determination of the crystalline volume fraction from the Raman spectra of microcrystalline silicon involves the knowledge of a material parameter called the Raman emission cross-section ratio $y$. This value is still debated in the literature. In the present work, the determination of $y$ has been carried out on the basis of quantitative analysis of medium-resolution transmission electron microscopy (TEM) micrographs performed on one layer deposited by very high frequency plasma enhanced chemical vapor deposition (VHFPECVD) close to the amorphous/microcrystalline transition. Subsequent comparison of these data with the crystallinity as evaluated from measured Raman spectra yields a surprisingly high value of $y=1.7$. This result is discussed in relation to previously published values (that range from 0.1 to 0.9 ).
\end{abstract}

(C) 2006 Elsevier B.V. All rights reserved.

PACS: 78.30.Ly; 78.67.Bf

Keywords: Raman scattering; STEM/TEM; TEM/STEM; Microcrystallinity

\section{Introduction}

The degree of crystallinity of hydrogenated microcrystalline silicon $(\mu \mathrm{c}-\mathrm{Si}: \mathrm{H})$ is an important measure for both layer and device characterization. Indeed, several material quality parameters such as defect density, dark- and photoconductivity and device properties such as open-circuit voltage $\left(V_{\mathrm{oc}}\right)$ depend mainly on the degree of crystallinity of the microcrystalline silicon intrinsic layer. The commonly used experimental technique for the quantification of crystallinity in microcrystalline silicon is Raman spectroscopy. Indeed, it is a desktop, fast and non-destructive measurement technique. In a Raman spectrum, the amorphous silicon phase of the $\mu \mathrm{c}-\mathrm{Si}: \mathrm{H}$ leads to the appearance of a broad peak centered at $480 \mathrm{~cm}^{-1}$, whereas the nanocrystalline silicon phase leads to the occurrence of an asymmetric peak centered at $520 \mathrm{~cm}^{-1}$. The low-wavenumber tail of this peak (around $510 \mathrm{~cm}^{-1}$ ) is attributed to the

\footnotetext{
* Corresponding author. Tel.: +41 3271832 00; fax: +41 327183201.

E-mail address: Evelyne.Vallat@unine.ch (E. Vallat-Sauvain).
}

defective but yet crystalline part of the nanocrystals. Integrated intensities of both phases $\left(I_{\mathrm{a}}\right.$ and $I_{\mathrm{c}}$, respectively) can be easily evaluated from the Raman spectra, and their ratio yields the so-called 'Raman crystallinity factor' $\phi_{\mathrm{c}}[1]$. This value is not identical with the actual crystalline volume fraction $X_{\mathrm{c}}$ which involves the additional parameter $y$ in the following way [2,3]: $X_{\mathrm{c}}=I_{\mathrm{c}} /\left(I_{\mathrm{c}}+y I_{\mathrm{a}}\right)$ where $y$ is the 'Raman emission cross-section ratio'.

The value of $y$ is at present a matter of debate. Values from 0.88 down to 0.1 have been published $[2,3]$. Furthermore, $y$ basically depends on the size of the crystallites and on the excitation wavelength used for the Raman experiment [2]. Thus, Raman emission cross-section ratio values for the amorphous and nanocrystalline phases are not at all known, with the necessary precision, for a quantitative evaluation of the crystallinity in $\mu \mathrm{c}-\mathrm{Si}: \mathrm{H}$.

Here, we report on quantitative analysis of the amorphous/microcrystalline phases present in $\mu \mathrm{c}-\mathrm{Si}: \mathrm{H}$ from transmission electron microscopy (TEM) micrographs and their relationship with the measured Raman crystallinity factors. From this comparison, a value is obtained for $y$. 
The value obtained for the Raman emission cross-section ratio in this work is higher than those previously published. It is, however, shown to be in agreement with the values obtained from three further reference samples: a highly microcrystalline and fully amorphous layer and from a monocrystalline silicon wafer.

\section{Experimental}

The sample used for the evaluation the parameter $y$ is a $\mu \mathrm{c}-\mathrm{Si}: \mathrm{H}$ layer deposited by VHF-PECVD near the $\mu \mathrm{c}-\mathrm{Si}: \mathrm{H} /$ a-Si:H transition, with a silane concentration of $\mathrm{SC}=6 \%$. This sample was chosen as it presents a microstructure consisting of a mix between amorphous and microcrystalline phases. Raman spectra were obtained in a commercial Renishaw Raman microscope in the back-scattering configuration using both a $\mathrm{HeNe}$ laser (excitation wavelength $633 \mathrm{~nm}$ ) and an Ar laser (excitation wavelength $514 \mathrm{~nm}$ ) in the bifacial mode [1]. The three peaks were deconvoluted with a commercial software (GRAMS) assuming a Gaussian shape. As discussed before, the $510 \mathrm{~cm}^{-1}$ contribution, attributed to the defective part of the crystalline phase, is included in the crystalline phase. Therefore, $\phi_{\mathrm{c}}$ will be calculated as follow:

$\phi_{\mathrm{c}}=I_{(\mu) \mathrm{c}} /\left(I_{(\mu) \mathrm{c}}+I_{\mathrm{a}}\right)=\left(I_{520}+I_{510}\right) /\left(I_{520}+I_{510}+I_{480}\right)$,

where $I_{i}$ is the area under the Gaussian peak centred at $i$ and $I_{520}+I_{510}+I_{480}$ is the total integrated intensity. According to $[2,3]$ the Raman emission cross-section ratio is

$y=\Sigma_{(\mu) \mathrm{c}} / \Sigma_{\mathrm{a}}$,

where $\Sigma_{(\mu) c}$ is the Raman emission cross-section of the material giving rise to the $520 \mathrm{~cm}^{-1}$ and $510 \mathrm{~cm}^{-1}$ peaks in the Raman spectra and $\Sigma_{\mathrm{a}}$ is the Raman emission cross-section of the amorphous silicon giving rise to the $480 \mathrm{~cm}^{-1}$ peak.

The sample for the TEM observation was prepared as cross-section with the 'tripod' mechanical thinning technique described in [4]. The TEM cross-section was then observed in a Philips CM200 microscope in the dark-field mode (medium-resolution) at $200 \mathrm{kV}$. Several TEM micrographs were taken, altogether yielding a representative sampling length of about $15 \mu \mathrm{m}$ in the plane parallel to the substrate.

\section{Results}

A typical cross-section micrograph of the sample studied here is shown in Fig. 1(a). From the micrographs of the sample cross-section, we have drawn and digitized two grey levels (white and black) pictures from the micrograph of the amorphous and microcrystalline phases (see Fig. 1(b), white and black parts, respectively). From these projected views of the thinned sample, it is possible to obtain the volume fraction of the respective amorphous and microcrystalline components of the structure [5]. For this purpose, we have measured at various depths $(z)$, on a one pixelthick line, the proportion of black pixels compared to the total number of pixels. This has been done with a commercial image processing software (analySIS, version 3.2). One can, thus, obtain a 'linear microcrystalline fraction' $C(z)$, represented in Fig. 2 from the bottom of the sample (fully amorphous, $C(z)=0$ ) to the first micrometer of the layer (fully microcrystalline, $C(z)=1$ ).

\section{Discussion}

In the Raman experiment, the volume of the sample probed by the excitation beam is proportional to the double optical attenuation function $A(z)=\exp (-2 \alpha z)$ where $\alpha$

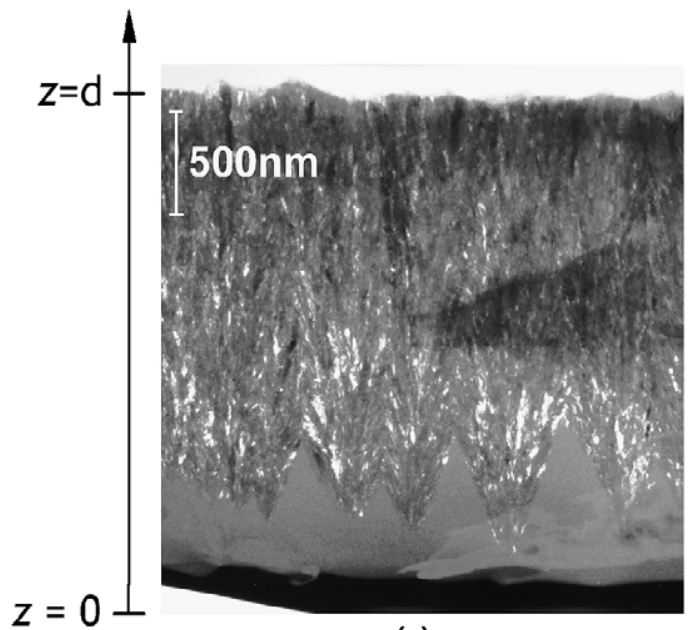

(a)

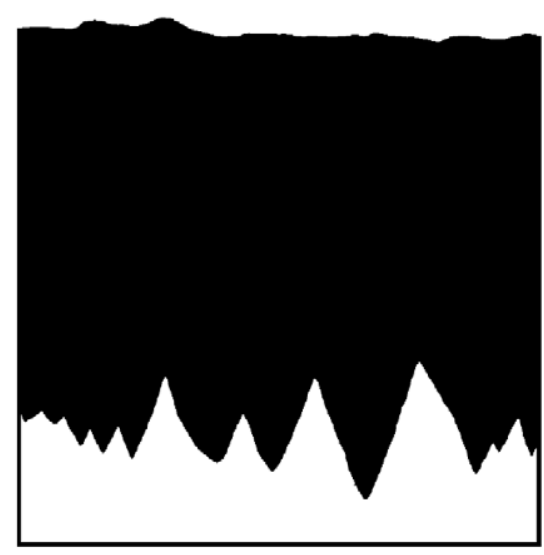

(b)

Fig. 1. (a) TEM dark-field cross-section of a $\mu \mathrm{c}-\mathrm{Si}: \mathrm{H}$ layer on glass and (b) its corresponding digitized micrograph with the amorphous (white) and microcrystalline (black) phases. 


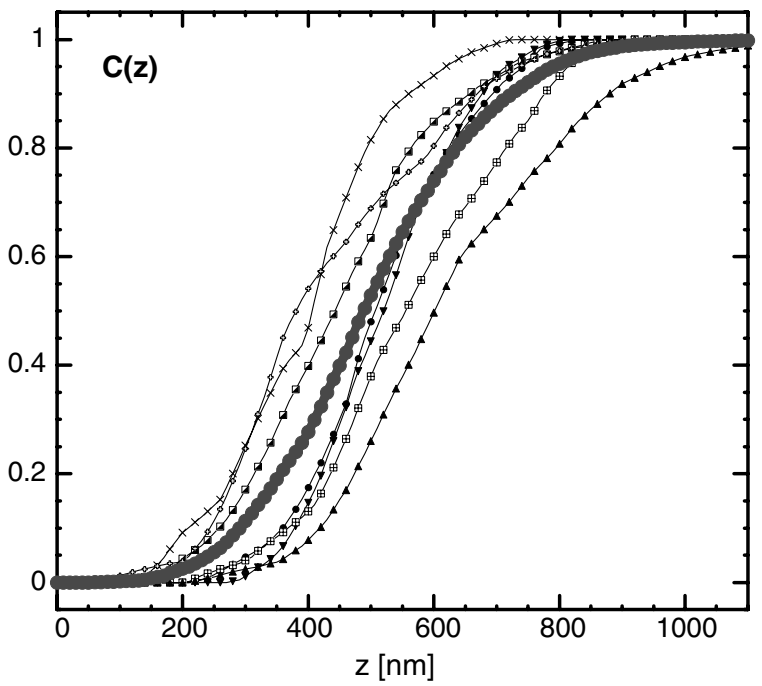

Fig. 2. Evolution of the linear microcrystallinity $C(z)$ with the sample depth. $Z=0$ at the glass-layer interface (bottom of the micrograph). The thick line is the average value of the seven $C(z)$ curves measured on different locations on the cross-section sample (thin lines).

is the absorption coefficient [6] and thus the $\mu \mathrm{c}-\mathrm{Si}: \mathrm{H}$ phase volume fraction within the Raman-probed volume is given by

$X_{\mu \mathrm{c}}=\frac{V_{\mu \mathrm{c}}}{V_{\mathrm{tot}}}=\frac{\int_{0}^{d}[A(z) \cdot C(z)]}{\int_{0}^{d} A(z)}$.

The parameter $X_{\mu \mathrm{c}}$ gives the average material crystallinity within the probed volume as if the microcrystalline phase (black on the digitized micrograph) had a crystalline volume fraction equal to 1 . But in reality, this is not the case as fully microcrystalline samples always contain some percentage of amorphous phase (e.g., within grain boundaries). Thus, we have to correct the parameter $X_{\mu \mathrm{c}}$ in order to take into account that the microcrystalline phase (within the cones, i.e., the black phase in the digitized micrograph) has a crystallinity lower than 1 . This is the reason why we introduce $\xi$ as the real crystalline fraction of the $\mu \mathrm{c}-\mathrm{Si}: \mathrm{H}$ phase, and suppose that $\xi$ is a constant within the sample (i.e., the crystalline fraction within the microcrystalline cones is not a function of $z$ ). Then real crystalline volume fraction as probed in the Raman experiment becomes

\section{Table 1}

Experimental values of $\phi_{\mathrm{c}}$ obtained from deconvoluted bifacial depthdependent Raman measurements, as well as calculated values for $X_{\mu \mathrm{c}}$ (with Eq. (3))

\begin{tabular}{lllll}
\hline & $\begin{array}{l}633 \mathrm{~nm}, \\
\text { layer-side }\end{array}$ & $\begin{array}{l}633 \mathrm{~nm}, \\
\text { glass-side }\end{array}$ & $\begin{array}{l}514 \mathrm{~nm}, \\
\text { layer-side }\end{array}$ & $\begin{array}{l}514 \mathrm{~nm}, \\
\text { glass-side }\end{array}$ \\
\hline$\phi_{\mathrm{c}}$ & 0.70 & 0.33 & 0.72 & 0.00 \\
$X_{\mu \mathrm{c}}$ (average) & 0.984 & 0.38 & 1 & $0.00-0.08$ \\
\hline
\end{tabular}

The absorption coefficient values used in Eq. (3) are (a) for a-Si:H: $10^{5} \mathrm{~cm}^{-1}$ at $514 \mathrm{~nm}$ and $10^{4} \mathrm{~cm}^{-1}$ at $633 \mathrm{~nm}$ and (b) for $\mu \mathrm{c}-\mathrm{Si}: H$ equal to $3 \times 10^{4} \mathrm{~cm}^{-1}$ at $514 \mathrm{~nm}$ and $10^{4} \mathrm{~cm}^{-1}$ at $633 \mathrm{~nm}$.
$X_{\mathrm{c}}=\xi \cdot X_{\mu \mathrm{c}}$.

And one can thus express $y$ as

$y=\frac{\phi_{\mathrm{c}}\left(1-X_{\mathrm{c}}\right)}{X_{\mathrm{c}}\left(1-\phi_{\mathrm{c}}\right)}=\frac{\phi_{\mathrm{c}}\left(1-\xi \cdot X_{\mu \mathrm{c}}\right)}{\xi \cdot X_{\mu \mathrm{c}}\left(1-\phi_{\mathrm{c}}\right)}$.

For our $\mu \mathrm{c}-\mathrm{Si}: \mathrm{H}$ layer, the experimental values of $\phi_{\mathrm{c}}$ as well as the calculated values for $X_{\mu \mathrm{c}}$ (for the average curve) are given in Table 1.

The value of $y$ can now be calculated with Eq. (5). However, we have first to find the value for $\xi$. We assert that the correct value for $\xi$ is that for which $y_{\text {glass-side }}=y_{\text {layer-side, }}$, at a given wavelength. As can be seen from Table 1, the strongly absorbed light $(514 \mathrm{~nm})$ is not useful for the determination of $y$, as the trivial value $\phi_{\mathrm{c}}=0$ for the glass-side is obtained. This value is due to the presence at the glasslayer interface of a fully amorphous incubation layer (see Fig. 1(a)). We have plotted in Fig. 3 the values of $y$ as a function of $\xi$ (according to Eq. (5)) for the values of $\phi_{\mathrm{c}}$ and $X_{\mathrm{\mu c}}$ given in Table 1 . For the average $C(z)$ curve (see Fig. 2$), \quad y(\xi)_{\text {glass-side }}=y(\xi)_{\text {layer-side }}$ when $\xi \approx 0.59$ and $y_{633} \approx 1.68$. As the crystalline volume fraction within the microcrystalline phase $\xi$ is obviously independent of the wavelength, the values for $y$ at $514 \mathrm{~nm}\left(y_{514}\right)$ can be obtained on the layer-side curve (see Fig. 3) for the values of $\xi$ obtained for $633 \mathrm{~nm}$. This leads to the a value of $y_{514} \approx 1.78$. These values for $y$ are surprisingly quite different than those ranging from 0.1 to 0.88 that were published previously $[2,3]$.

As a countercheck, one can obtain the Raman emission cross-section ratios from the comparison of the Raman intensity measured for monocrystalline silicon wafer with that obtained from a fully amorphous sample, thus yielding

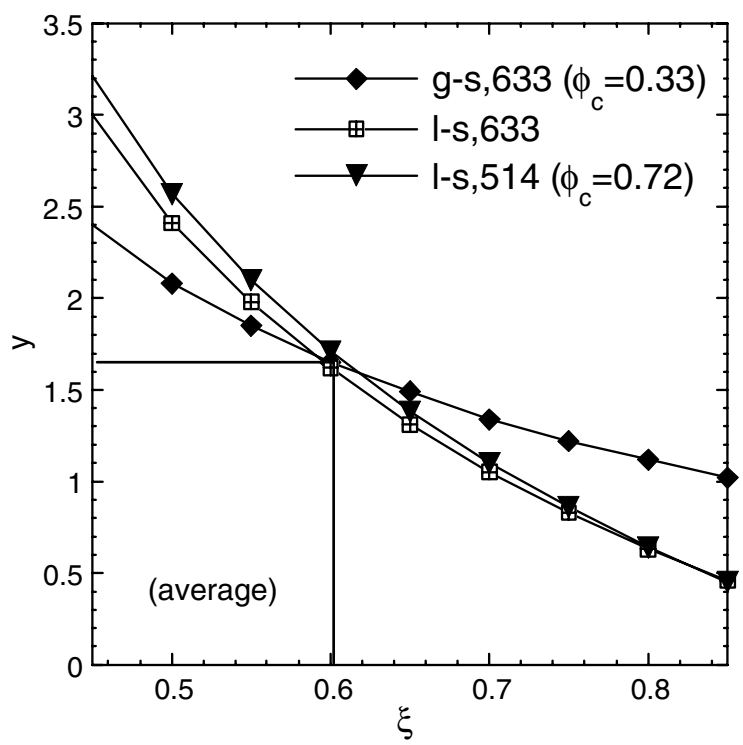

Fig. 3. Relation between the Raman cross-section ratio $y$ and $\xi$ calculated with Eq. (4) from the Raman crystallinity factors $\phi_{\mathrm{c}}$ and the $X_{\mu \mathrm{c}}$-values given in Table 1. The correct values for $y$ and $\xi$ are those obtained where the curves for the glass-side (g-s) measurement cross that for the layer-side (1-s) measurement. Therefore, $y_{633}=1.68$ and $y_{514}=1.78$ for $\xi=0.59$. 
$y^{\mathrm{c} / \mathrm{a}}$. The comparison of the Raman intensity measured for a fully microcrystalline sample with that obtained for a fully amorphous layer will yield $y_{514}^{\mu \mathrm{c} / \mathrm{a}}$. The integrated Raman intensities obtained on such samples must be corrected with the respective absorption coefficients [2]

$y=\frac{\sum_{(\mu) \mathrm{c}}}{\sum_{\mathrm{a}}} \approx \frac{I_{(\mu) \mathrm{c}}}{I_{\mathrm{a}}} \cdot \frac{\alpha_{(\mu) \mathrm{c}}}{\alpha_{\mathrm{a}}}$.

By using the absorption coefficients $\alpha$ given in Table 1 for the respective materials, one obtains $y^{\mu \mathrm{c} / \mathrm{a}} \approx 2.1-2.8$ (a value close to the $y_{514}$-value measured in our sample) and $y^{\mathrm{c} / \mathrm{a}} \approx 0.4-1.1$ (a value in the range of the previously published values). The difference between $y_{514}^{\mu \mathrm{c} / \mathrm{a}}$ and $y_{514}^{\mathrm{c} / \mathrm{a}}$ thus obtained can explain (at least in part) the difference between the value of $y$ evaluated above $(y \approx 1.7-1.8)$ and the values found in the literature $(y \approx 0.1-0.9)$.

\section{Conclusion}

The ratio of Raman emission cross-sections has been determined experimentally from the quantitative analysis of volume fractions of the amorphous and microcrystalline phases, as observed in transmission electron micrographs.
By comparison of this value with the measured Raman crystallinity factor, a ratio $y=1.7$ of emission cross-sections has been obtained. Our determination of $y$ allows now for a more accurate evaluation of the actual crystalline fraction in microcrystalline silicon.

\section{Acknowledgment}

We acknowledge financial support from the Swiss 'Office Fédéral de l'Energie (OFEN)' under contract No. 36487 as well as from the Swiss National Science Foundation under grant FN66985.

\section{References}

[1] C. Droz et al., Sol. Energy Mater. Sol. Cells 81 (2004) 61.

[2] E. Bustarret et al., Appl. Phys. Lett. 52 (1988) 1675.

[3] M.H. Brodsky et al., Phys. Rev. B 16 (1977) 3556;

R. Tsu et al., Appl. Phys. Lett. 40 (1982) 534.

[4] J. Benedict et al., Mater. Res. Symp. Proc. 254 (1992) 121.

[5] U. Underwood, Quantitative Stereology, Addison-Wesley, Reading, MA, 1970, p. 175.

[6] Z. Hang et al., J. Appl. Phys. 64 (1988) 3233. 